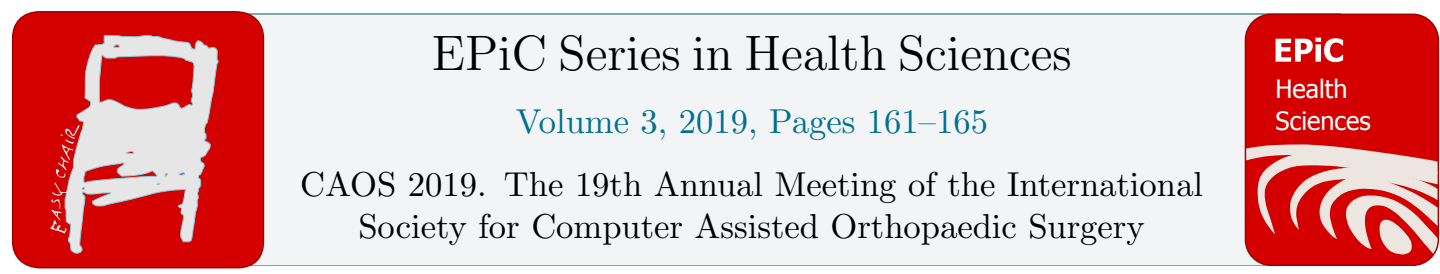

\title{
Patient-specific ROM and load-based Target Zone for Total Hip Arthroplasty and its Application in a retrospective Analysis
}

\author{
Juliana Habor ${ }^{1}$, Maximilian C. M. Fischer ${ }^{1}$, Kunihiko Tokunaga ${ }^{2}$, Masashi \\ Okamoto $^{3}$, Klaus Radermacher ${ }^{1}$ \\ ${ }^{1}$ Chair of Medical Engineering, Helmholtz Institute for Biomedical Engineering, RWTH Aachen \\ University, Germany \\ ${ }^{2}$ Niigata Hip Joint Center, Kameda Daiichi Hospital, Niigata City, Japan \\ ${ }^{3}$ Department of Radiology, Kameda Daiichi Hospital, Niigata City, Japan \\ j.habor@hia.rwth-aachen.de
}

\begin{abstract}
The outcome of total hip arthroplasty (THA) depends on multiple alignment and design parameters. Unsuitable parameter settings could lead to impingement, dislocation, increased wear, and loosening. This work introduces a method for calculating a patient-specific target zone based on range of motion (ROM) related and load related criteria. Possible bone or prosthesis impingement are analyzed. The resulting hip force is calculated and compared to the pre-operative situation. The edge loading risk is analyzed. Pelvic tilt is considered for the calculation of the ROM and the load. THA parameters fulfilling all criteria are included in the target zone.

The above described method has been applied to 30 cases retrospectively. All cases had been planned and navigated on the basis of CT data. From each patient, pre- and post-operative CT and EOS data and Harris Scores were acquired. The pre-operative data served as the input data for the target zone calculator. We hypothesized that cases with post-operative THA parameters inside the target zones have higher scores than other cases.

The patients whose implants are within the target zones had higher scores than the remaining patients. Especially patients inside both target zones (combined target zone) had higher scores. The results also show that for 19 out of 30 patients, conventional CT-based planning and navigation does not provide optimal placement regarding the combined target zone. A further validation of the method with a larger sample size is part of our ongoing work.
\end{abstract}

\section{Introduction}

The outcome of total hip arthroplasty (THA) depends on multiple alignment and design parameters. Unsuitable parameter settings could lead to impingement, dislocation, increased wear, and loosening. In clinical routine, standard values such as the Lewinnek safe zone [Lewinnek et al. 
1978] and the combined anteversion technique [Ranawat \& Maynard 1991; Dorr et al. 2009; Weber et al. 2016] are often used for cup alignment. However, studies show that dislocations occur even if the cup is placed in the suggested target zone [Callanan et al. 2011; Abdel et al. 2016]. The reason might be that the target zones are statistically derived values for the average patient. A more patient-specific adaptation of these values could improve the outcome for the individual patient.

The prosthesis has to fulfil certain constraints that might be contradictory to each other. Impingement of both the prosthesis and the bones should be avoided while providing a sufficient range of motion (ROM) and the resulting hip force should not be too high in amplitude and not at the edge of the cup causing edge loading. A method for calculating a patient-specific target zone incorporating ROM-based and load-based criteria was developed and tested on 30 cases retrospectively.

\section{Materials and Methods}
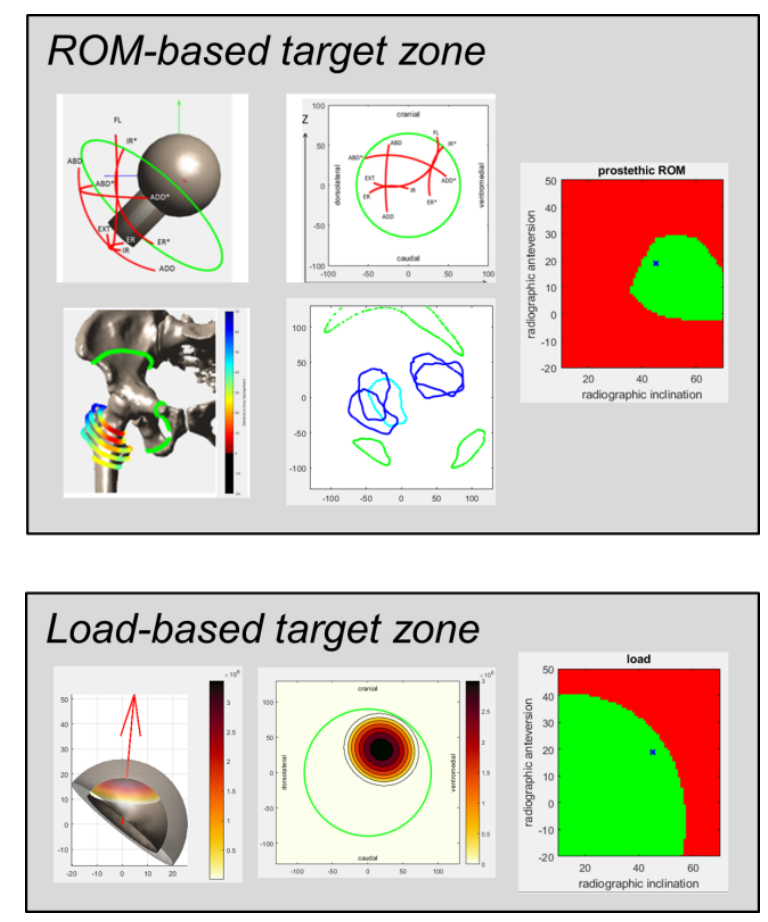

Figure 1: Calculation of the patient-specific ROM-based and load-based THA target zones.

The concept for calculating the combined target zone is shown in Figure 1. Single target zones are calculated regarding the patient specific ROM and resulting hip load conditions. The ROM-based target zone, including all impingement free cup orientations, depends on the stem design, its orientation and the head-to-neck ratio [Hsu et al. 2019]. The distance to bony impingement is also calculated [Hsu et al. 2018] and compared to the pre-operative situation. The ROM suggested by Yoshimine [Yoshimine 2006] is used as the target ROM. The load-based target zone depends on the position of the muscle attachment points relative to the center of rotation and is influenced by the stem design and orientation as well as the cup position. The direction of the resulting hip force is calculated using a modified Iglic model [Iglic et al. 1990]. The model was adapted to the pelvic width, pelvic height and the position of the greater trochanter. The contact patch is calculated using the 
Imado model [Imado et al. 2005]. The load-based target zone includes all cup orientations without unsuitable edge loading that would occur when the contact patch overlaps with the rim of the cup. Furthermore, the resulting hip force should not be larger than the pre-operative value. The cup orientation is defined relative to the anterior pelvic plane. Pelvic tilt is considered for the calculation of the ROM and the load.

The above described method has been applied to 30 cases retrospectively. All cases had been planned and navigated on the basis of CT data. From each patient, pre- and post-operative CT and EOS data and Harris Scores [Harris 1969] were acquired. The pre-operative data served as the input data for the target zone calculator. We hypothesized that cases with post-operative THA parameters inside the target zones have higher scores than other cases.

\section{Results}

The results are shown in Figure 2. The patients whose implants are within the target zones had higher scores than the remaining patients. Especially patients inside both target zones (combined target zone) had higher scores.
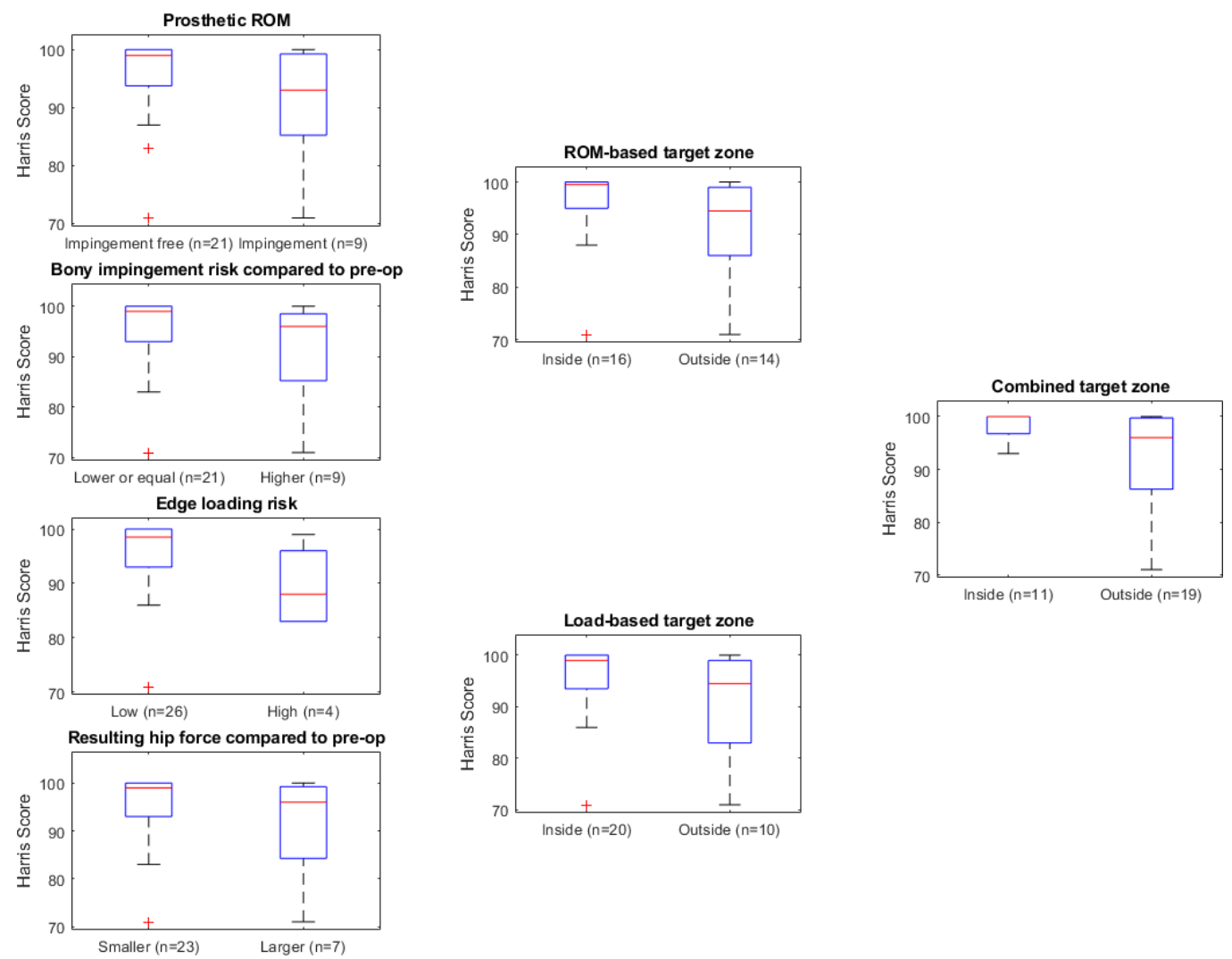

Figure 2: Comparison of post-operative Harris scores of patients inside and outside of target zones. 


\section{Discussion}

Finding optimal THA parameters is the topic of numerous studies but a common safe zone could not be identified [Seagrave et al. 2017; Snijders et al. 2018]. Most of the studies compared dislocated with non-dislocated hips. Other studies derive a safe zone based on a ROM analysis and suggested rules for the combined anteversion [Widmer \& Zurfluh 2004; Yoshimine 2006]. Weber et al. found that these rules are useful for detecting prosthetic impingement but do not prevent bony impingement [Weber et al. 2016]. Incorporating load-related factors is also of importance and was addressed in several studies [Pierrepont et al. 2013; Elkins et al. 2015; Clarke \& Lazennec 2015]. The results of our study suggest that THAs which are classified as within the patient-specific target zones are associated with higher outcome scores. The results also show that for 19 out of 30 patients, conventional CT-based planning and navigation does not provide optimal placement regarding the combined target zone.

There are certain limitations in this preliminary study and potentials for improvement for the method. A further validation of the method with a larger sample size is part of our ongoing work. Although each target zone is based upon published models, the validity of each target zone has to be further investigated. Some of the data had osteophytes around the native acetabulum. This could have an influence on the calculated cup coverage and bony impingement risk. Related improvements are subject to our ongoing work.

\section{References}

[Abdel et al. 2016] Abdel, M. P; Roth, P. von; Jennings, M. T; Hanssen, A. D; Pagnano, M. W.: What safe zone? The vast majority of dislocated THAs are within the Lewinnek safe zone for acetabular component position. In: Clinical Orthopaedics and Related Research, 474 (2016), 2: 386-391

[Callanan et al. 2011] CALlanan, M. C; JarRett, B; Bragdon, C. R; Zurakowski, D; Rubash, H. E; FreiberG, A. A; MalchaU, H.: The John Charnley Award: risk factors for cup malpositioning: quality improvement through a joint registry at a tertiary hospital. In: Clinical Orthopaedics and Related Research, 469 (2011), 2: 319-329

[Dorr et al. 2009] DorR, L. D; MALIK, A; DASTANE, M; WAN, Z.: Combined anteversion technique for total hip arthroplasty. In: Clinical Orthopaedics and Related Research, 467 (2009), 1: 119-127

[Harris 1969] HARRIS, W. H.: Traumatic Arthritis of the Hip after Dislocation and Acetabular Fractures: Treatment by Mold ArthroplastyAN END-RESULT STUDY USING A NEW METHOD OF RESULT EVALUATION. In: The Journal of bone and joint surgery. American volume, 51 (1969), 4: 737-755

[Hsu et al. 2019] Hsu, J; DE LA Fuente, M; RADERMACHER, K.: Calculation of impingement-free combined cup and stem alignments based on the patient-specific pelvic tilt. In: Journal of Biomechanics, 82 (2019): 193-203

[Hsu et al. 2018] Hsu, J; Fischer, M. C. M; Tokunaga, K; Esnault, G; Radermacher, K.: Analyzing Bony Constraints as a Key Stone of an Integrated Approach Towards Functional THA Planning. In: Wei Tian \& Rodriguez Y Baena, F. (eds.): CAOS 2018. EPiC Series in Health Sciences: EasyChair (2018) (EPiC Series in Health Sciences): 74-78

[Iglic et al. 1990] Iglic, A; SraKar, F; Antolic, V; Kralu-Iglic, V; BatagelJ, V.: Mathematical analysis of Chiari osteotomy. In: Acta Orthop Iugosl, 20 (1990): 35-39

[Imado et al. 2005] IMADO, K; KIDO, Y; MIYAGAWA, H.: A method of calculation for contact pressure between femoral head and cup of artificial hip joint. In: Tribology Transactions, 48 (2005), 2: $230-237$ 
[Lewinnek et al. 1978] LeWINNEK, G. E; LeWIS, J. L; TARR, R; COMPERE, C. L; ZiMMERMAN, JR: Dislocations after total hip-replacement arthroplasties. In: The Journal of Bone \& Joint Surgery, 60 (1978), 2: 217-220

[Ranawat \& Maynard 1991] Ranawat, C. S. \& MAYNARD, M. J.: Modern technique of cemented total hip arthroplasty. In: Techniques in Orthopaedics, 6 (1991), 3: 17-25

[Weber et al. 2016] Weber, M; Woerner, M; Craiovan, B; Voellner, F; WorliceK, M; SPRINGORUM, H.-R; GRIFKA, J; RENKAWITZ, T.: Current standard rules of combined anteversion prevent prosthetic impingement but ignore osseous contact in total hip arthroplasty. In: International Orthopaedics, 40 (2016), 12: 2495-2504

[Yoshimine 2006] Yoshimine, F.: The safe-zones for combined cup and neck anteversions that fulfill the essential range of motion and their optimum combination in total hip replacements. In: Journal of Biomechanics, 39 (2006), 7: 1315-1323

\section{Disclosures}

This work has been supported in parts by ConforMIS, Inc., Billerica, USA. 